\title{
Corrigendum
}

\section{Modelling income protection claim termination rates by cause of sickness, Parts I and II - CORRIGENDUM}

\author{
By S. Y. Ling, H. R. Waters and A. D. Wilkie
}

Part I - doi:10.1017/S1748499500000713, Published by Cambridge University Press, 10 May 2010

Part II - doi:10.1017/S1748499500000725, Published by Cambridge University Press, 10 May 2010

Part III - doi:10.1017/S1748499500000737, Published by Cambridge University Press, 10 May 2010

In a series of three papers, Ling et al. (2009a, 2009b \& 2009c), the authors set out formulae for the calculation of the recovery and mortality intensities for Income Protection Insurance (IPI) claimants. These formulae, which allow for duration of sickness, age, cause of claim and other factors, are, unsurprisingly, complex. Regrettably, there are some typographical errors in the published formulae. This short note corrects these errors and provides some clarification regarding the calculation of the intensities. The errors in the original papers were all typographical and did not affect the numerical results or the conclusions presented in those papers.

The corrections/clarifications all relate to Papers I and II and are set out in Paper/Section order.

\section{Contact address}

Sing Yee Ling, Ernst \& Young, Ten George Street, Edinburgh, EH2 2DZ, U.K. Tel: 0044 (0)131 777 2000. E-mail: SLing@UK.EY.com

\section{Paper I}

\subsection{Paragraph 4.3}

The definition of $x_{\text {year }}$ should be:

$$
x_{\text {year }}=(\text { Year }-1988) / 16
$$

\subsection{Table 4}

There is no need to include $\alpha_{D P 1}$ as a retained covariate for the three models since DP1 is the baseline category for deferred period and hence is included by default in the three models.

$\alpha_{a g e 2}$ is not a retained covariate in the model for $c s 36$. See Table 6. 


\subsection{Paragraph 4.5}

The duration of sickness, $z$, is measured in years but the break points, for example $\tau_{D P 4}$, are expressed in days, with 365 days in each year. Also, in formulae (iii) and (iv), the $i-$ th power is taken after taking the positive part. Hence, the correct illustrative formulae for (i), (iii) and (iv) should be:

(i) $\gamma_{D P 4}\left(\tau_{D P 4}-365 z\right)_{+}$

(iii) $\zeta_{D P 4_{i}}\left[\left(C_{1}\left(t_{k}\left(\tau_{D P 4} / 365\right)\right)-C_{1}\left(t_{k}(z)\right)\right)_{+}\right]^{i}, \quad i=1,2, \ldots$

(iv) $\phi_{D P 4_{i}}\left[\left(C_{1}\left(t_{k}(z)\right)-C_{1}\left(t_{k}\left(\tau_{D P 4} / 365\right)\right)\right)_{+}\right]^{i}, \quad i=1,2, \ldots$

Formula (ii) is unchanged.

For example, the terms to be included in the model for $\operatorname{cs} 32, \operatorname{cs} 33, \operatorname{cs} 34$, cs 37 and $\operatorname{cs} 38$ should be:

$$
\begin{aligned}
& \beta_{\text {year }}(z)=\alpha_{\text {year }}+\theta_{\text {year }} C_{1}\left(t_{k}(z)\right) \\
& \beta_{D P 4}(z)=\alpha_{D P 4}+\gamma_{D P 4_{1}}\left(\tau_{D P 4_{1}}-365 z\right)_{+}+\gamma_{D P 4_{2}}\left(\tau_{D P 4_{2}}-365 z\right)_{+} \\
& \beta_{D P 13}(z)=\alpha_{D P 13}+\gamma_{D P 13}\left(\tau_{D P 13}-365 z\right)_{+}
\end{aligned}
$$

where $\tau_{D P 4_{1}}=37.5, \tau_{D P 4_{2}}=70.5$ and $\tau_{D P 13}=125.5$.

As a further example, the term $\sum_{i=1}^{n} x_{\text {agei }} \beta_{\text {agei }}(z)$ to be included in the model for cause $\operatorname{cs} 27$ is:

$$
\begin{aligned}
\sum_{i=1}^{n} x_{\text {agei }} \beta_{\text {agei }} & =x_{\text {age } 1} \beta_{\text {age } 1}(z)+x_{\text {age } 2} \beta_{\text {age } 2}(x) \\
\text { where } x_{\text {agei }} & =C_{i}((\text { Age }-43) / 26) \\
\beta_{\text {age } 1}(z) & =\alpha_{\text {age }}+\zeta_{\text {age } 1}\left(C_{1}\left(t_{k}\left(\tau_{\text {age }} / 365\right)\right)-C_{1}\left(t_{k}(z)\right)\right)_{+} \\
\beta_{\text {age } 2}(z) & =\alpha_{\text {age } 2}+\zeta_{\text {age } 2}\left[\left(C_{1}\left(t_{k}\left(\tau_{\text {age }} / 365\right)\right)-C_{1}\left(t_{k}(z)\right)\right)_{+}\right]^{2}
\end{aligned}
$$

See Table 11 for the parameter values.

\subsection{Paragraph 4.6}

For the calculation of the baseline hazard, $\rho_{0}(z)$, the duration of sickness, $z$, is measured in years.

\subsection{Paragraph 4.7}

Formula (3) should include within the second exponential a term:

$$
x_{\text {cause }} \beta_{\text {cause }}
$$

since, where several causes are being modelled within the same sickness category, each cause may have its own cause parameter. This is the case for causes cs32, cs33, cs34 and cs37 in Category G6, with $c s 38$ being the baseline cause for this group. 
Formula (3) contains the terms $\sum_{i=1}^{n} x_{\text {yeari }} \beta_{\text {yeari }}$ and $\sum_{i=1}^{n} x_{\text {agei }} \beta_{\text {agei }}$. Note that, as explained in Section 4.3: $x_{\text {yeari }}$ is $C_{i}\left(x_{\text {year }}\right)$, where $C_{i}()$ is the Chebycheff polynomial of order $i$ and $x_{\text {year }}=($ Year -1988$) / 16$, and,

$x_{\text {agei }}$ is $C_{i}\left(x_{\text {age }}\right)$, where $C_{i}()$ is the Chebycheff polynomial of order $i$ and $x_{\text {age }}=(x-43) / 26$.

\subsection{Figure 6}

The caption and legend for Figure 6 should specify that the recovery intensities for cs 36 are for males. This is not required for $\operatorname{cs} 38$ since for this cause the recovery intensities do not depend on sex.

\subsection{Paragraph 5.1}

The coefficient -0.2885 in the second formula in point (iv) should be -0.2881 . The final result, 0.7646 , is unchanged.

The final two paragraphs of point $(\mathrm{v})$ has some numerical errors; in particular, the denominator in the factor for Year should be 16 rather than 13 . Hence these paragraphs should be:

For cs36 we can adjust the intensities so that they relate to other years by multiplying by a factor $\exp (-0.4584($ Year-1988)/16). For example, intensities for the calendar year 1975 would be a multiple 1.4513 and intensities for 2002 would be a multipe 0.6696 of those shown in Figure 6. This deterioration in recovery rates over the range of our data is consistent with the values in CMIR18 (2000, Table 4A) and CMIR22 (2005, Table 4A) for all causes combined and for deferred periods greater than one week. The recovery intensities for 2011, which is well beyond the range of our data, would be a multiple 0.5174 of those shown in Figure 6. Whether this continued deterioration is realistic is beyond the scope of this paper. A pragmatic approach for anyone requiring recovery intensities applicable to years later than 2002 might be to use the 2002 rates as produced by our models, but, given the deterioration in these rates over the period 1975 to 2002, caution would need to be exercised.

For cs38 the Year adjustment is a function of the duration of sickness $z$ (in years), given by

$$
\exp \left(\left(0.5774-9.4564 \frac{z}{1+6.7 z}\right)\left(\frac{\text { Year }-1988}{16}\right)\right)
$$

Hence, for $z=26$ weeks and for calendar year 2002, the recovery intensities for both DP1 and DP4 would be a multiple 0.6407 of those shown in Figure 6.

\subsection{Table 11}

The value of the parameter $\alpha_{D P 13}$ is zero so that the formula for $\beta_{D P 13}$ is:

$$
\beta_{D P 13}=-0.0094 \times(198.5-365 z)
$$

\subsection{Table 14}

For cause 50 , the parameter $\tau_{D P 4_{1}}$ has the value 43.5 days. 


\section{Paper II}

\subsection{Paragraph 4.3}

The final model for the mortality of male assured lives presented in Paper II (formula (5)) was:

$$
\log \lambda_{x y}=\sum_{i=0}^{6} b_{i} C_{i}\left(x^{\prime}\right)+\sum_{i=1}^{4}\left(\beta_{i}+\sum_{j=1}^{5} \gamma_{i j} w_{j}\left(x^{\prime}\right)\right) C_{i}\left(y^{\prime}\right)
$$

This is incorrect. The correct formula is:

$$
\log \lambda_{x y}=\sum_{i=0}^{6} b_{i} C_{i}\left(x^{\prime}\right)+\sum_{i=1}^{4}\left(\beta_{i}+\sum_{j=1}^{5} \gamma_{i j} w_{i j}\left(x^{\prime}\right)\right) C_{i}\left(y^{\prime}\right)
$$

The difference is that there are separate spline functions for the age-dependent coefficients of the year adjustment, labelled $i=1,2,3,4$. Each of these splines has six knots and the knots are placed as shown in Table 1.

Table 1. Placement of knots for the splines for the age-dependent coefficients.

\begin{tabular}{lllllll}
\hline \hline$i=$ & $\tau_{1}$ & $\tau_{2}$ & $\tau_{3}$ & $\tau_{4}$ & $\tau_{5}$ & $\tau_{6}$ \\
\hline 1 & 23.5 & 36.1 & 48.7 & 61.3 & 73.9 & 86.5 \\
2 & 23.5 & 36.1 & 48.7 & 61.3 & 73.9 & 86.5 \\
3 & 21.75 & 50.25 & 59.75 & 69.25 & 78.75 & 88.25 \\
4 & 23.5 & 45.5 & 55 & 65 & 67.5 & 88.25 \\
\hline \hline
\end{tabular}

\subsection{Paragraph 5}

The final model for the mortality of female assured lives presented in Paper II (formula (7)) was:

$$
\begin{aligned}
\log \lambda_{x y}= & \sum_{i=0}^{7} b_{i} C_{i}\left(x^{\prime}\right)+\beta_{1} w_{1}\left(y^{\prime}\right)+\beta_{2} w_{2}\left(y^{\prime}\right)+\sum_{i=3}^{4}\left(\beta_{i}+\gamma_{i 1} x^{\prime}+\sum_{j=2}^{3} \gamma_{i j} w_{j}\left(x^{\prime}\right)\right) w_{i}\left(y^{\prime}\right) \\
& +\left(\beta_{5}+\gamma_{51} x^{\prime}+\gamma_{52}\left(x^{\prime}-(76-55) / 35\right)_{+}\right) w_{5}\left(y^{\prime}\right)
\end{aligned}
$$

This is incorrect. The correct formula is:

$$
\begin{aligned}
\log \lambda_{x y}= & \sum_{i=0}^{7} b_{i} C_{i}\left(x^{\prime}\right)+\beta_{1} w_{1}\left(y^{\prime}\right)+\beta_{2} w_{2}\left(y^{\prime}\right)+\sum_{i=3}^{4}\left(\beta_{i}+\sum_{j=1}^{3} \gamma_{i j} w_{i j}\left(x^{\prime}\right)\right) w_{i}\left(y^{\prime}\right) \\
& +\left(\beta_{5}+\gamma_{51} x^{\prime}+\gamma_{52}\left(x^{\prime}-(76-55) / 35\right)_{+}\right) w_{5}\left(y^{\prime}\right)
\end{aligned}
$$

The authors regret any inconvenience caused by the errors in the original papers and are grateful to Andrew Stott for identifying many of the errors.

\section{References}

Ling, S.Y., Waters, H.R. \& Wilkie, A.D. (2009a). Modelling Income Protection Claim Terminations by Cause of Sickness I: Recoveries. Annals of Actuarial Science, 4(Part II), 199-239; doi:10.1017/S1748499500000713. 
Ling, S.Y., Waters, H.R. \& Wilkie, A.D. (2009b). Modelling Income Protection Claim Terminations by Cause of Sickness II: Mortality of UK Assured Lives. Annals of Actuarial Science, 4(Part II), 241-259; doi:10.1017/S1748499500000725.

Ling, S.Y., Waters, H.R. \& Wilkie, A.D. (2009c). Modelling Income Protection Claim Terminations by Cause of Sickness III: Excess Mortality. Annals of Actuarial Science, 4(Part II), 261-286; doi:10.1017/S1748499500000737. 